\title{
Intervenciones para optimizar la adherencia y la capacidad para la toma de medicamentos en adultos mayores polimedicados
}

\author{
Interventions to optimize adherence in older adults prescribed with multiple medications
}

\section{Comentado de:}

Cross A J, et al. Cochrane Database Syst Rev. 2020, 5:CD012419. PMID: $32383493^{1}$

\section{Objetivo}

Evaluar la efectividad de intervenciones diseñadas para mejorar la capacidad de toma de medicación y/o la adherencia en adultos mayores que viven en la comunidad a quienes se les prescribieron múltiples medicamentos a largo plazo.

\section{Diseño}

Revisión sistemática.

\section{Métodos de búsqueda}

Los autores realizaron búsquedas en las siguientes bases de datos electrónicas: Cochrane Central Register of Controlled Trials (CENTRAL), MEDLINE, Embase, PsycINFO, Cumulative Index to Nursing and Allied Health Literature (CINAHL) e International Pharmaceutical Abstracts (IPA), desde su inicio hasta junio de 2019. También realizaron búsquedas en la literatura gris y en los registros de ensayos en línea hasta noviembre de 2017, y revisaron las listas de referencias de los estudios incluidos.

\section{Selección de estudios}

Fueron incluidos ensayos controlados aleatorizados (ECA), ensayos controlados cuasialeatorizados (quasi-ECA) y ensayos controlados aleatorizados por conglomerados (cluster-ECA), que hubieran comparado un grupo de personas asignadas a una intervención para mejorar la capacidad de toma de medicación, o la adherencia a la toma de la misma, con un grupo asignado a la atención habitual (ninguna intervención) o a una intervención diferente.

Los participantes de los estudios primarios debían tener 65 años de edad o más, vivir en su hogar y tomar cuatro o más medicamentos. Las intervenciones fueron clasificadas en tres grupos según sus componentes: educativas, conductuales o mixtas. Los estudios que habían valorado la adherencia en un plazo menor a cuatro semanas y los que habían evaluado la capacidad de toma de medicación en un período menor a 48 horas fueron excluidos.

\section{Extracción y análisis de los datos}

Dos autores, de forma independiente, extrajeron los datos, evaluaron e informaron el riesgo de sesgo de los estudios incluidos. Las discrepancias fueron resueltas mediante el consenso con un tercer autor.

Los desenlaces primarios fueron la habilidad o capacidad para tomar la medicación y la adherencia a la medicación. La capacidad de toma de los medicamentos fue definida como la competencia de una persona para seguir con precisión un régimen de medicamentos recetado (conocer qué medicamentos tomar, cuándo tomarlos y poder administrarlos correctamente). La adherencia a los medicamentos fue definida como la medida en que la conducta de toma de los medicamentos se corresponde con las recomendaciones de tratamiento acordadas con el proveedor de atención médica.

Los desenlaces secundarios incluyeron el conocimiento del paciente $y / 0$ de su cuidador acerca de sus medicamentos, la satisfacción de ambos respecto de la intervención, la calidad de vida relacionada con la salud, algunos desenlaces clínicos adversos para la salud (p. ej., consultas a departamentos de emergencias, visitas al médico de cabecera o efectos adversos), otros desenlaces específicos de cada condición clínica en particular ( $p$ ej., glucemia), la mortalidad y la costo efectividad de la intervención evaluada.

El metanálisis fue realizado, cuando fue posible, utilizando un modelo de efectos aleatorios de acuerdo a las recomendaciones Cochrane. La certeza general de la evidencia para cada resultado fue evaluada utilizando los criterios GRADE.

\section{Resultados}

Esta revisión incluyó 50 estudios, de los cuales 40 fueron ECA, cuatro quasi-ECA y seis cluster-ECA, con un total de 14.269 participantes. La Tabla 1 muestra los resultados sobre la adherencia a la medicación, que contó con información proveniente de 14 estudios primarios (7 incluidos en el meta-análisis) que evaluaron intervenciones educativas, 7 estudios (4 incluidos en el metaanálisis), intervenciones conductuales, y 27 estudios (19 incluidos en el meta-análisis), intervenciones mixtas. Para evaluar la capacidad de toma de la medicación los autores no encontraron estudios de intervenciones educativas y conductuales. Sólo un ensayo documentó mejoras significativas en un puntaje de comportamiento para las intervenciones mixtas $(86 / 100$ vs $74 / 100$; $p=0,001$, baja calidad de evidencia).

Las intervenciones conductuales y educativas parecen tener un bajo o nulo beneficio en la calidad de vida relacionada a la salud. Si bien las intervenciones mixtas produjeron una disminución en el riesgo de internación en los 11 estudios meta analizados (riesgo relativo [RR]) fue 0,67 , con intervalo de confianza (IC) del IC $95 \% 0,5$ a 0,9 ), la calidad de la evidencia fue baja y con alto grado de heterogeneidad entre los estudios ( $\left.{ }^{2} 73 \%\right)$. La mortalidad en los pacientes estudiados no se vio impactada por las intervenciones mixtas (RR 0,93, IC 95\% 0,67 a 1,30, muy baja calidad de evidencia). Para el resto de los desenlaces e intervenciones evaluadas la evidencia recuperada fue escasa y de baja calidad.

\section{Conclusiones}

Un estudio de intervención mixta -con baja calidad de evidencia- documentó una mejoría en la capacidad de toma de medicamentos. Las intervenciones conductuales y las mixtas pueden mejorar la proporción de personas que adhieren satisfactoriamente a los medicamentos recetados, mientras que el efecto de las exclusivamente educativas fue beneficioso sólo al ser evaluado de manera dicotómica (evidencia de muy baja certeza).

Se necesitan más ECA de alta calidad metodológica que evalúen los efectos de las intervenciones para mejorar la capacidad de toma de medicamentos y la adherencia a la medicación, así como también otras variables de interés sanitario, en los adultos mayores a los que se prescriben múltiples medicamentos.

Fuente de financiamiento / Conflicto de interés de los autores: Dos autoras fueron apoyadas por becas del Programa de Capacitación en Investigación del Gobierno de Australia durante su participación en esta revisión. 
Tabla 1. Adherencia a la medicación: síntesis de los resultados para cada tipo de intervenciones analizadas en la revisión. Nota: IC: intervalo de confianza.

\begin{tabular}{|l|c|c|c|}
\hline Intervenciones & Riesgo Relativo (IC 95\%) & Diferencia de Medias Estandarizada (IC 95\%) & Certeza de la evidencia \\
\hline Educativa & $1,66(1,33$ a 2,06$)$ (2 estudios) & $0,16(-0,12$ a 0,43$)(5$ estudios $)$ & Muy baja \\
\hline Conductual & $1,22(1,07$ a 1,38$)(4$ estudios $)$ & No evaluada & Muy baja \\
\hline Mixta & $1,22(1,08$ a 1,37$)(12$ estudios $)$ & $0,47(-0,08$ a 1,02$)(7$ estudios $)$ & Baja \\
\hline
\end{tabular}

\section{Comentario}

Las personas mayores de 65 años tienen con frecuencia múltiples problemas de salud crónicos, por lo que resultan expuestas a la polifarmacia (uso de cuatro o más medicamentos) ${ }^{2}$. Cuando esta se asocia a dificultades físicas y cognitivas para la toma de medicación de manera correcta, podría aumentar el riesgo reacciones adversas, el uso de tratamientos en dosis subóptimas y la sobreutilización de medicamentos ${ }^{3-5}$. De manera adicional, la prescripción de regímenes terapéuticos complejos, el seguimiento inadecuado por los profesionales de la salud y el apoyo social deficiente podrían incrementar la utilización de los servicios sanitarios, las hospitalizaciones y la morbimortalidad ${ }^{6}$.

En este contexto, en 2003 la Organización Mundial de la Salud reconoció la importancia de mejorar la adherencia a la toma de medicamentos como estrategia para abordar de manera eficaz las enfermedades crónicas. La capacidad para tomar la medicación y la adherencia son además necesarias para garantizar la seguridad y la efectividad del uso de medicamentos. Si bien estos conceptos son ampliamente utilizados por el equipo de salud, existe variabilidad en los significados atribuidos a estos términos en diferentes ámbitos de atención y especialidades médicas o no médicas.

De acuerdo a las definiciones empleadas en la revisión resumida, la no adherencia es pensada como un fenómeno dicotómico que puede ser involuntario o intencional. En el primer caso las causas subyacentes involucran la falta de comprensión de la indicación, los problemas físicos y/o la complejidad del régimen prescrito. En el segundo, la persona decide no tomar la medicación según las instrucciones recibidas o acordadas con el equipo de salud. Este marco podría ser cuestionado desde la perspectiva de la atención centrada en las personas, ya que el término "no adherencia voluntaria" connota cierto grado de paternalismo al asumir que sólo el profesional de la salud puede y debe decidir cuál es la mejor prescripción para su paciente, y si el paciente no cumple con la recomendación pone en riesgo su salud.

No obstante la rigurosa metodología utilizada en la elaboración de esta revisión, resulta evidente que fenómenos como la adherencia son por demás complejos e involucran diversos factores facilitadores y obstaculizadores que deberían ser revisados en profundidad en próximos textos. Sin embargo, se entiende que las variables utilizadas en el encuadre de la medicina basada en la evidencia deben simplificarse para poder abordar soluciones prácticas.

\section{Conclusiones de la comentadora}

Las estrategias educativas para aumentar la capacidad de toma de medicamentos de los adultos mayores no cuentan aún con buen respaldo bibliográfico. Tampoco hay evidencia concluyente que avale las intervenciones educativas, conductuales o mixtas para los desenlaces de mayor relevancia clínica, como las hospitalizaciones o la mortalidad. Las intervenciones analizadas podrían mejorar la adherencia, aunque la evidencia es de baja confianza. Cabe destacar la simplicidad con la que fue definida esta variable tan compleja y multicausal, que debería ser abordada con mayor profundidad en futuros estudios.

María Agustina Vega [ Servicio de Medicina Familiar y Comunitaria, Hospital Italiano de Buenos Aires. agustina.vega@hospitalitaliano.org.ar ]

Vega MA Intervenciones para optimizar la adherencia y la capacidad para la toma de medicamentos en adultos mayores polimedicados. Evid Actual Pract Ambul. 2021;24(4):e002150. Available from: https://dx.doi.org/10.51987/EVIDENCIA.V25I1.6990. Comentado de: Cross A J, et al. Interventions for improving medication-taking ability and adherence in older adults prescribed multiple medications.Cochrane Database Syst Rev. $2020,5: C D 012419$. PMID: 32383493. doi:10.1002/14651858.cd012419.pub2

\section{Referencias}

1. Cross AJ, Elliott RA, Petrie K, et al. Interventions for improving medication-taking ability and adherence in older adults prescribed multiple medications. Cochrane Database Syst Rev. 2020;5(5):CD012419. Available from: 10.1002/14651858.CD012419.pub2.

2. Patterson SM, Cadogan CA. Interventions to improve the appropriate use of polypharmacy for older people. Cochrane Database Syst Rev. 2014;7(10):CD008165. Available from: 10.1002/14651858.CD008165.pub3

3. Barbas NR, Wilde EA. Competency issues in dementia: medical decision making, driving, and independent living. J Geriatr Psychiatry Neurol. 2001;14(4):199-212. Available from: 10.1177/089198870101400405.

4. Beckman A, Bernsten C, Parker MG, et al. The difficulty of opening medicine containers in old age: a population-based study. Pharm World Sci. 2005;27(5):393-398. Available from: 10.1007/s11096-005-7903-z.

5. Leendertse AJ, Egberts ACG, Stoker LJ, et al. Frequency of and risk factors for preventable medication-related hospital admissions in the Netherlands. Arch Intern Med. 2008;168(17):1890-1896. Available from: 10.1001/archinternmed.2008.3.

6. Howard RL, Avery AJ, Howard PD, et al. Investigation into the reasons for preventable drug related admissions to a medical admissions unit: observational study. Qual Saf Health Care. 2003;12(4):280-285. Available from: 10.1136/qhc.12.4.280. 